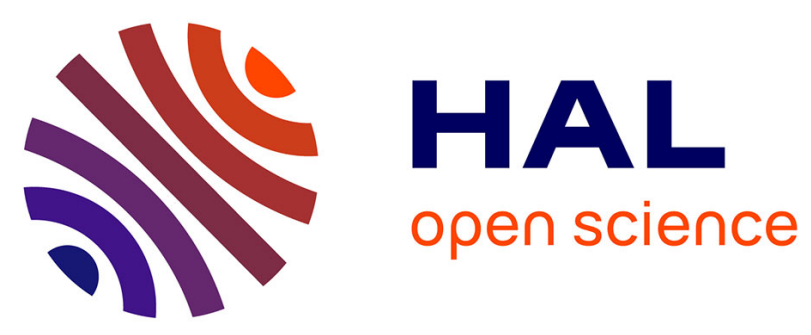

\title{
Sensory Feedback and Interactivity: Enhancing Motivation and Engagement for VR Stroke Rehabilitation
}

Fatin Shamimi Mohd Zuki, Suziah Sulaiman, Dayang Rohaya Awang Rambli, Frederic Merienne, Mohamad Naufal Mohamad Saad

\section{To cite this version:}

Fatin Shamimi Mohd Zuki, Suziah Sulaiman, Dayang Rohaya Awang Rambli, Frederic Merienne, Mohamad Naufal Mohamad Saad. Sensory Feedback and Interactivity: Enhancing Motivation and Engagement for VR Stroke Rehabilitation. 2021 International Conference on Computer \& Information Sciences (ICCOINS), Jul 2021, Kuching, Malaysia. pp.333-338, 10.1109/iccoins49721.2021.9497200 . hal-03336877

\section{HAL Id: hal-03336877 https://hal.science/hal-03336877}

Submitted on 7 Sep 2021

HAL is a multi-disciplinary open access archive for the deposit and dissemination of scientific research documents, whether they are published or not. The documents may come from teaching and research institutions in France or abroad, or from public or private research centers.
L'archive ouverte pluridisciplinaire $\mathbf{H A L}$, est destinée au dépôt et à la diffusion de documents scientifiques de niveau recherche, publiés ou non, émanant des établissements d'enseignement et de recherche français ou étrangers, des laboratoires publics ou privés. 


\section{Sensory Feedback and Interactivity: Enhancing Motivation and Engagement for VR Stroke Rehabilitation}

\author{
Fatin Shamimi Mohd Zuki \\ Computer and Information Sciences \\ Department \\ Universiti Teknologi PETRONAS \\ Tronoh, Malaysia \\ fatin_18003397@utp.edu.my \\ Frédéric Merienne \\ Institut Image - ENSAM \\ Arts et Métiers ParisTech \\ Chalon-sur-Saône, France \\ frederic.merienne@ensam.eu
}

\author{
Suziah Sulaiman \\ Computer and Information Sciences \\ Department \\ Universiti Teknologi PETRONAS \\ Tronoh, Malaysia \\ suziah@utp.edu.my
}

Mohamad Naufal Mohamad Saad

Computer and Information Sciences Department

Universiti Teknologi PETRONAS

Tronoh, Malaysia

naufal_saad@utp.edu.my

\author{
Dayang Rohaya Awang Rambli \\ Computer and Information Sciences \\ Department \\ Universiti Teknologi PETRONAS \\ Tronoh, Malaysia \\ dayangrohaya.ar@utp.edu.my
}

\begin{abstract}
Stroke is a condition that happens when the brain is cut off from blood supply. Patients are at risk of disabilities. To help patient recover, gain mobility, and independence, rehabilitation starts as soon as possible. Unfortunately for some patients, they are forced to undergo long-term rehabilitation period. Since the activities during therapy are repetitive, many patient losses their motivation to continue therapy thus unable to recover. Virtual reality has been found to increase motivation for stroke rehabilitation. Its key elements, especially sensory feedback and interactivity have been found to increase motivation and engagement especially when applied to gamification. However, practitioners in related field have raised concern on the relevancy of some VR elements used for rehabilitation. Inappropriate elements may not be effective for the program; thus, requires further investigation. This paper explores various elements that could encourage motivation, bring better engagement, and enhance task performance of stroke patients via VR-based rehabilitation. A literature survey was conducted. The findings signal for the importance of sensory feedback and interactivity when used in VR environment for stroke patients in their rehabilitation programme.
\end{abstract}

Keywords-Virtual reality, sensory feedback, stroke, rehabilitation

\section{INTRODUCTION}

A cut off of blood supply to the brain due to blockage by a clot or ruptures of the arteries leads to a stroke. Brain damage caused by stroke usually affects one side of the brain, either on the right or left hemisphere. The side of the damaged brain will cause disability on the opposite side of the body.

Often, disabilities caused by stroke are long-term disabilities. When this happens patients lose their independence and would require a long-term care. Rehabilitation is important for the recovery of stroke patients due to loss of mobility and other functions. It helps patients relearn skills lost and most importantly to improve function so patients can regain their independence. The process of rehabilitation is to treat most of function lost, improving the functional and intellectual capacities from neuroplasticity, and re-educating motor cognitive functions [1], [2].

There are various approaches of therapies in stroke rehabilitation including physical activities, and cognitive and emotional activities. Physical activities involve motor-skill exercises, mobility training, and constraint-induced therapy. Cognitive and emotional activities are therapy for cognitive disorders, communication disorders, psychological evaluation and treatment, and medication. The speed of recovery differs by the severity and other complications patient may face. This affects the duration of stroke rehabilitation.

Prolonged rehabilitation can cause motivational issues in patients. As time goes by, patients are inclined to get tired of the therapy exercises and would consider the training program as monotonous [3]. Frequent training with high intensity of repetitions turn rehabilitation into a mundane and depressing task for the patients. As the patients get tired and bored of the training process, they will find themselves losing motivation for rehabilitation.

Other corresponding factors to the problem of rehabilitation is patients' engagement in the therapy sessions. Engagement has been defined by [4] in their study as patients' intentional effort to work toward recovery by participating fully in rehabilitation therapies. The study revealed that the most frequently rated encountered barriers impacting patient engagement are fear of pain, depressed mood, and cognitive issues. These issues damper the motivation of patients to start or continue with rehabilitation. In the end, patients will be noncompliant with rehabilitation program. Motivation and engagement of patients in receiving rehabilitation is also interlinked with task performance of the patients. It has been concluded by [5] that highly repetitive training can result in improved recovery. Repetition combined with active engagement by the patients undergoing rehabilitation encourages reorganization.

To keep patients undergoing rehabilitation interested and motivated, interactive and appealing set of training procedures should be introduced to patients. The trend has shown that interactive gaming being put forward for rehabilitation with the purpose of making training fun and contextual [6]. While interactive gaming for rehabilitation can be achieved through pc-based games and mobile applications, previous studies by [7], [8] showed that robot training using virtual reality (VR) has been revealed to enhance stroke rehabilitation. Preliminary studies by [9] also indicated the potential of VR as a tool for physical 
rehabilitation. It is then further proved that VR provides a unique medium that achieves the requirements for effective rehabilitation intervention [10]. VR application can provide therapy within a functional, purposeful, and motivating context. Bohil et al. [11] add that VR simulations can be highly engaging, providing crucial motivation for rehabilitative applications requiring consistent, repetitive practice. Furthermore, improvement of brain plasticity and regenerative processes is possible through real and ecological demands of the real world offered by VR [12].

Despite the potential benefits VR could bring to stroke rehabilitation, practitioners in related field have raised concern on the relevancy of some VR elements used for rehabilitation. Inappropriate elements may not be effective for the program [13]. It has been observed that less is being discussed on the relationship of VR key elements and theories of gamification involving incentives and rewards in supporting the rehabilitation. Without this understanding, the effectiveness of such a VR system may be difficult to evaluate. Questions that include (i) what constitutes in the VR elements that make stroke patients perform during rehabilitation, and (ii) how do the elements relate to patients' motivation and engagement are two fundamental issues that need investigation.

This paper aims to explore various elements that could encourage motivation, bring better engagement, and enhance task performance of stroke patients via VR-based rehabilitation. It presents a literature review on VR multisensory feedback, and engagement retention in relation to the feedback. The findings will be used for discussion purposes at the end of this paper.

\section{LITERATURE REVIEW}

\section{A. Key elements of virtual reality experience}

The popularity of VR could also be credited to how relatable and real a simulated situation or environment is when VR is used. Information now is no longer static and non-dimensional, which also means VR adds value to user experience. According to Sherman and Craig [14], there are four key elements of virtual reality experience $-a$ virtual world, immersion, sensory feedback, and interactivity.

How does VR allow engagement and motivation for rehabilitation for the patients? The four key elements provide a suitable environment exclusively for the rehabilitation that is immersive physically and mentally through the augmentation of the senses. The use of sensory feedback through various modalities such as the visual, auditory, and tactile feedback as well as interactive design of the rehabilitation system could add fun into the therapy session. Therefore, patients will be more motivated to go through therapy and will be more engaged in the session.

\section{B. Multi-sensory feedback in virtual reality}

Not only sensory feedback is an essential element in virtual reality experience, it also provides VR users with significant benefit. Sensory feedback stimulates brain centers due to CNS plasticity[15]. Bohil et al. remark that VR engages the sensorimotor system much more by providing realistic stimulation to multiple sensory channels simultaneously. One of the interesting issues in stroke rehabilitation using $\mathrm{VR}$ is the use of multi-sensory feedbacks.
Multi-sensory is the integration of different senses (sight, touch, hearing, smell, and taste), however, Navarro et al. [16] stated that vestibular or balance sense as part of hearing and proprioception is also a part of multi-sensory component. Feedback sent through multi-sensory channels in VR means that user can receive information not only visually but through other senses as well. The term multi-sensory and multimodality can also be used interchangeably as defined by Varni and Castagné [17]: a modality is identified as a perceptual modality while multimodality is identified as multi-sensory. Teo et al. [18] claim that VR environment produces multi-sensory feedback which in turn contributes toward better memory consolidation and retention. VR allows a sense of overall well-being due to the simulation of multiple channels by use of auditory and visual feedback which stimulate patient's awareness of his performance.

\section{Visual feedback}

In et al. [19] examined the effects of visual feedback with a mirror on stroke patients' balance ability. The outcome demonstrates benefits of visual feedback from a mirror for improving balance ability during quiet standing on an unstable surface in patients with stroke. Although the experiment was conducted outside of the VR domain, such result is a benchmark proving that visual feedback does improve task performance for stroke rehabilitation. However, [20] have another opinion on mirrors or videorecordings visual feedback. Although these methods provide patients with visual feedback of the movement performance and allow them to be aware and correct their movements, they are not optimal after a stroke due to cognitive and selfimage issues. The authors noted that when using these methods, viewpoints are often limited and aspect of movement sequences that would benefit from observation in different planes are failed to be captured. From the patient point of view, they can be distracted by the clinical background or even distressed by their appearance post stroke. Additionally, difficulties such as views being obscured by clothing and problems with differentiating left from right among stroke patients due to lowered cognitive function when looking at mirror reflection are some of the weaknesses of mirror therapy.

The use dynamic visualizations of the patient's movements have been suggested as able to facilitate gait reeducation [20]. A stick figure representing the patient was shown on a monitor and mimics the patient's movement in either real time or after the completion of an action depending on the tasks and the patient's capability. A follow up of the study [21] revealed improvement in walking velocity, step length, spatial symmetry, and temporal symmetry. The study found that the participants were able to engage with the avatar and expressed that they found it helpful to be able to see how they moved. With VR, environments and scenarios can be created according to the ideal situation with lesser distraction.

\section{Auditory feedback}

Audio could also act as an effective medium in a VR stroke rehabilitation program as there are strong correlation between music and motor functions; music aids motor learning [22]. Researches [23], [24] found strong plasticity effects on motor brain areas from long- and short-term musical practice. Rosati et al. [25] supports the findings through reviewing studies in neurosciences. Many of the studies suggest that auditory stimulation can improve brain 
plasticity by affecting specific mechanisms that contribute significantly to recovery from neurological damage.

According to Sihvonen et al. [26], the scope of formal music-based intervention can be active intervention or receptive intervention. Active intervention includes activities such as creating music, playing an instrument, singing, or musical improvisation. On the other hand, receptive intervention involves listening to music that is administered by a credentialed music therapist. Sihvonen et al. reviewed sixteen randomized controlled trials that used music as a component of therapy for stroke-related neurological and neuropsychiatric disturbances. From the review, eight studies revealed rehabilitation with a musicbased intervention enhanced motor recovery in stroke patients. In one of the studies [28], the improvement in motor skills was found to be specifically caused by music rather than by motor training alone as patients practicing with mute instruments showed less improvement. The study by Cha et al. shows improvement in balance, gait velocity, cadence, stride length, and double support period on the affected side on the group that underwent movement therapy with rhythmic music accompaniment. These findings indicate that auditory feedback does add value on task performance of the patients.

Besides improving task performance, Särkämö et al. [15] recommend listening to music to enhance cognitive recovery and mood in post-stroke patients. Other than that, auditory feedback such as music might bring better engagement towards the rehabilitation process of stroke patients. As found by Forsblom et al. [16], music listening provides better relaxation, increased motor activity, and improved mood. Not only does auditory feedback improve mood hence elevating the motivation of patients to undergo rehabilitation, Rosati et al. [12] point out that informative feedback on errors in movement can sustain motivation during learning. Aspects of persuasion can be applied as auditory feedback to elevate motivation during therapy. Application of human-computer dialogue support such as praises, suggestions, and walkthrough may brighten patient's mood and keep the engagement level high.

\section{E. Haptic feedback}

Haptic feedback is classified into two classes: kinesthetic feedback and tactile feedback. Simply put, kinesthetic feedback can be felt from sensors of the muscles, joints, and tendons. This kind of feedback gives the brain ability to approximate size, weight, and position of and object relative to the body. Meanwhile, tactile feedback can be felt in fingers or on the surface. The sensors for sensing tactile feedback are embedded in the skin and right beneath it. Such feedback allows brain to process feels such as vibration, pressure, touch, and texture. Kinesthetic feedback will be beneficial for gross motor therapy while tactile feedback will benefit fine motor therapy in stroke rehabilitation using VR. In VR, haptic feedback is usually presented through wearables such as haptic gloves or full body suits.

As claimed by Hoffman et al. [17], tactile augmentation is an effective technique for adding texture and force feedback to virtual objects. With haptic feedback in VR, the participants are more engaged in the virtual world. They had more fun, found the event more realistic, and showed slight inclination to feel more present in the virtual world. Besides, Weber et al. [18] recommend active force feedback as it significantly enrich interaction in VR, resulting in higher sensation of presence and or immersion.

Other than engagement and motivation, haptic feedback is found to improve task performance of patients undergoing motor therapy using VR [19]. This complements the statement by [20] whom identified haptic as substantial for improving a patient's performance in more difficult tasks. A report by Shing et al. [21] demonstrated that haptic feedback is particularly beneficial when added to an upper extremity movement when the difficulty of the task was high. Findings by Weber et al. show that participant had better performance in term of precision, mental workload, and spatial orientation when assisted with force feedback as compared to vibrotactile and visual feedback.

\section{F. Engagement retention through multi-sensory feedback reward system}

Barett et al. [22], reviewed various game design principles and the theoretical grounding of its presence. These principles could be utilized in the creation of stroke rehabilitation systems. The study shows that games can be a powerful therapeutic tool when intelligently integrated into rehabilitation to combat concerns such as low motivation and engagement. Core factors in game design such as meaningful play, feedback, goals, rewards, challenge, difficulty, failure and flow could be integral to the creation of custom rehabilitative system or games as it is to commercial games.

Widmer et al. [23] suggested the use of monetary reward as a motivation in rehabilitation after stroke. The result of the trial done by Widmer et al. demonstrated positive outcome on the motivation of patients in rehabilitation. However, the brain process compliment is equivalent of a social reward as being rewarded with money [24]. This finding was made through investigating the effect of praise on motor skill consolidation; the study found that participants that receive praises for their performance indicated improvement in their motor skills memory. Even though Widmer et al. used money as reward to promote motivation, the authors suggest that other forms of reward such as social rewards (smiles, praises), food rewards (sweets, dietary allowance), or token programs may be applied in rehabilitation. Reward can be presented to patients in the form of multisensory feedbacks in VR based on the core factors in game design as previously mentioned.

Ricther et al. [25] summarized the theoretical base of incentives and rewards in gamification as shown in Table 1. Personal investment theory is represented by PIT in the table.

\section{G. Existing Literature on VR-based Stroke Therapy}

A systematic search was conducted on IEEE Xplore, Elsevier (Science Direct), and Scopus journal databases in January to September 2019. The year of publications is set for 5 years from 2015 to 2019 due to the evolutionary nature of technology especially virtual reality. This search is to explore various virtual reality-based rehabilitation design which increase patient's engagement level in rehabilitation for stroke patient.

The search yielded 30 articles, of which 19 are potentially relevant papers to be retrieved in full text for further evaluation. Then, the 19 articles are screened by contents which excludes criteria such as: article is general literature on technology-based stroke rehabilitation, article is a clinical practice or a guideline, research does not include 
Table 1. Theoretical base of incentives and rewards in gamification

\begin{tabular}{|c|c|c|}
\hline Motivation Theory & Incentives/ Rewards & Role \\
\hline \multirow[t]{2}{*}{ Self-efficacy } & $\begin{array}{l}\text { Audio/verbal/ } \\
\text { visual/music/ } \\
\text { sound effect }\end{array}$ & Feedback \\
\hline & Progress bar & Feedback, achievements \\
\hline \multirow{2}{*}{$\begin{array}{l}\text { Self-efficacy, } \\
\text { goal-setting, PIT, expectancy value, } \\
\text { need achievement }\end{array}$} & $\begin{array}{l}\text { Points/bonus/ } \\
\text { dividend }\end{array}$ & $\begin{array}{l}\text { Feedback, reward, status, achievements, competition, progression, } \\
\text { ownership }\end{array}$ \\
\hline & $\begin{array}{l}\text { Mini games/ } \\
\text { challenges/quests }\end{array}$ & Reward, status, competition, achievements \\
\hline \multirow[t]{4}{*}{$\begin{array}{l}\text { Self-efficacy, goal-setting, PIT, expectancy value, } \\
\text { social comparison }\end{array}$} & Badges & $\begin{array}{l}\text { Status and reputation, achievements and past accomplishments, collection, } \\
\text { competition, ownership }\end{array}$ \\
\hline & Virtual goods & Reward, social, status, achievements, ownership, self-expression \\
\hline & Leaderboard & Status and reputation, achievements, competition \\
\hline & $\begin{array}{l}\text { Rewards- } \\
\text { choosing colours, } \\
\text { power }\end{array}$ & Achievements \\
\hline \multirow{2}{*}{$\begin{array}{l}\text { Self-efficacy, } \\
\text { goal-setting, PIT, expectancy value, } \\
\text { need achievement, social comparison }\end{array}$} & Achievements & Collection, status, competition, discovering, progression \\
\hline & Levels & $\begin{array}{l}\text { Feedback, status and reputation, achievements, competition, moderate } \\
\text { challenge }\end{array}$ \\
\hline $\begin{array}{l}\text { Social comparison, personal investment theory, } \\
\text { expectancy value }\end{array}$ & Avatar & Social, self-expression, ownership \\
\hline
\end{tabular}

any visual, haptic, or auditory modalities in rehabilitation programme, no theories for design of VR-based stroke rehabilitation, engagement level is not increased. The findings are tabulated in table 2 .

\section{DISCUSSION}

From the literature, it is found that the itself supports a better engagement and higher motivation through its multisensory feedbacks and interactivity. Studies also found that sensory feedback stimulates brain centers due to CNS plasticity. The processing of sensory feedback and knowledge of results allows cognitive acquisition of and memorization of movements. Thon [26] stated that generally, the association of cognitive process effects motor learning positively. Thus, the inclusion of sensory feedback to stroke rehabilitation might increase task performance of stroke patients undergoing therapies.

There are many ways to design the interactivity of VR, but the most popular technique is through gamification. Coincidentally, the core factors in game design have been found to support rehabilitation. Gamification factor such as rewards and feedbacks are included in the theoretical base of incentives and rewards. In this theory, each motivation goal has its own design and role. Hence, it is possible to target the type of motivation that is needed to be boosted for rehabilitation purposes during design and development of system. Interactivity could also integrate multi-sensory feedback as the input and output to increase patients' engagement. Besides, the incentives and rewards could be presented in these modalities, for example, points and praises through audio and visual cues.

Based on this theory, multi-sensory feedback in VRbased stroke rehabilitation system encourage motivation, bring better engagement, and enhance task performance of stroke patients.
Beside theoretical studies, there are also existing studies with prototypes developed for VR-based stroke rehabilitation as shown in table 2. The studies are able to prove that VR, gamification, and some sensory modalities indeed enhanced motivation and engagement. Unfortunately, there are lack of emphasize on the type of modalities used in the experiments.

This research topic lacks studies on how much of the relative weight of each sensory feedback (visual, auditory, or haptic) adds value to the whole experience during therapy. Although all modalities positively impact stroke rehabilitation, there could be modalities that are less effective than the others in aiding recovery. This proposition is in parallel with that highlighted by rehabilitation practitioners reported in [13]. The focus should no longer be on the usefulness of VR technology anymore but more specific on to the VR elements i.e. the relevancy and appropriateness of such elements for implementation. This shift of focus could be useful to address the lack of guidelines in the application of multi-sensory modalities in VR-based stroke rehabilitation system.

As shown in table 2, various studies have explored and proved the effectiveness of interactivity and multi-sensory feedback in rehabilitation via VR. However, most of studies, as pointed out earlier, lack details on why and how specific elements of sensory feedbacks are used. Most studies are limited in their findings of cognition, engagement, and motivation factor of their rehabilitation system. Furthermore, it was not explicitly shown that the selections of gameplay or pattern as well as the sensory feedbacks are user-centered. Inability to consider how users experience and use a system for rehabilitation might affect the effectiveness of the therapy by demotivating and disengaging users. This will override the effectiveness of sensory feedback and interactivity offered by VR, causing higher rate of noncompliance. 


\begin{tabular}{lll}
\hline $\begin{array}{l}\text { Barrett, Swain, } \\
\text { Gatzidis, \& } \\
\text { Mecheraoui (2016) }\end{array}$ & $\begin{array}{l}\text { Games can be a powerful therapeutic tool when intelligently integrated into } \\
\text { rehabilitation to combat concerns such as low motivation and low engagement. }\end{array}$ \\
{$[22]$} & $\begin{array}{l}\text { Core factors in game design such as meaningful play, feedback, goals, rewards, } \\
\text { challenge, difficulty, failure and flow could be integral to the creation of custom } \\
\text { rehabilitative games as it is to commercial games. Particularly, if these core factors } \\
\text { which are found to add to motivation, engagement and commercial success are } \\
\text { combined with technologically advanced control systems that compensate for motor } \\
\text { impairment. }\end{array}$
\end{tabular}

- $\quad$ Limited research elucidating these game design concepts and advocating their utilisation in stroke rehabilitation.

\section{Massetti et al. \\ (2018)}

Bai \& Song (2019) Yuan, Tsoupikova, \& Kamper (2018)

Pham, Phan,
Nguyen, \& Nguyen
(2016)

[30]
- Evidences from the review show the potentials of VR to have beneficial effects on rehabilitation of patients with neurological disorders.

- VR is reportedly to have psychological and cognitive benefits when adapted to the patient.

- $\quad$ Elderly patients using VR as rehabilitative device was found to have improvement in attention or memory stimulation.

- VR is likely to be an increasingly important option to complement traditional therapies.

- Hospital clinical trial results show that the system may increase patients' motivation in training.

- Varieties of rehabilitation training virtual scenes were added to execute rehabilitation training for patients and effectively improve their enthusiasm.

- There are needs for innovative, functional, and interactive VR devices that are available and accessible for individuals with such disorders.

- More studies are required to prove the effectiveness of existing VR technology.

- The study does not mention any auditory aspect of the games.

- It is not mentioned whether these games are able to deeply engage subjects with its visual display or possibly with any auditory support.

- No cognitive benefits of the games were discussed.

- The study does not mention any auditory aspect of the games.

- Cyclical stretching of the hand muscles through imposed joint rotation can reduce muscle hyper-excitability in stroke survivors.

- It also improves task performance with the impaired hand.

- Haptic feedback from the glove can aid sensorimotor learning.

- Rehabilitative games should motivate patients to exercise, should be repeated many times.

- $\quad$ Each session should be short and the different level of difficulty should be present

- Hand motions are able to be detected during therapy with game although low cost camera is used.
- $\quad$ No result of study with actual stroke patient using the game system.

- $\quad$ Lack of evidence showing the game pattern and the designed game helps in stroke rehabilitation.
From the literature, we have learnt that while sensory feedbacks are found to be effective and are widely studied, not much research have been focusing on which specific elements of the sensory feedbacks enhance motivation and engagement factors during rehabilitation. This is followed by sparse information and design model or framework on developing a VR-based rehabilitation system with multisensory feedback. On top of that, most studies are not design with a user-centered approach, thus not enough evidence showing that the users can still be compliant to use the systems in an extended duration.

\section{CONCLUSION AND FUTURE WORK}

This paper presented the literature of key elements of VR, multi-sensory feedbacks, and theories of gamification incentives and rewards in supporting the rehabilitation of stroke patients. The outcome of this study shows that these features have potential in assisting recovery. More importantly, the features are said to be able to encourage motivation, bring better engagement, and enhance task performance during rehabilitation.
Studies on the design and framework of rehabilitative games would be conducted in the future. Then the effectiveness and efficiency of each modality would be measured in order to obtain the hierarchy of the modalities for developmental guidelines.

\section{REFERENCES}

[1] W. R. Sherman and A. B. Craig, Understanding Virtual RealityInterface, Application, and Design, vol. 12, no. 4. 2003.

[2] L. Szmekova, J. Havelkova, and T. Katolicka, "The efficiency of cognitive therapy using virtual reality on upper limb mobility in stroke patients," in International Conference on Virtual Rehabilitation, ICVR, 2015, pp. 115-116, doi: 10.1109/ICVR.2015.7358599.

[3] E. Navarro, P. González, V. López-Jaquero, F. Montero, J. P. Molina, and D. Romero-Ayuso, "Adaptive, Multisensorial, Physiological and Social: The Next Generation of Telerehabilitation Systems," Front. Neuroinform., vol. 12, no. July, pp. 1-7, Jul. 2018, doi: 10.3389/fninf.2018.00043.

[4] G. Varni and N. Castagné, "Multimodal (multisensory) integration, in technology," in Enaction and enactive interfaces : a handbook of terms, E. S. Books, Ed. 2007, pp. 209-211. 
W.-P. Teo et al., "Corrigendum: Does a Combination of Virtual Reality, Neuromodulation and Neuroimaging Provide a Comprehensive Platform for Neurorehabilitation? - A Narrative Review of the Literature," Front. Hum. Neurosci., vol. 11, no. June, 2017, doi: 10.3389/fnhum.2017.00053.

T.-S. In, Y.-R. Cha, J.-H. Jung, and K.-S. Jung, "Effects of visual feedback with a mirror on balance ability in patients with stroke," J. Phys. Ther. Sci., vol. 28, no. 1, pp. 181-185, 2016, doi: 10.1589/jpts.28.181.

[7] H. Thikey, M. Grealy, F. van Wijck, M. Barber, and P. Rowe, "Augmented visual feedback of movement performance to enhance walking recovery after stroke: Study protocol for a pilot randomised controlled trial," Trials, vol. 13, no. 1, p. 1, 2012, doi: 10.1186/1745-6215-13-163.

[8] H. Thikey, F. van Wijck, M. Grealy, and P. Rowe, "A virtual avatar to facilitate gait rehabilitation post-stroke," Gait Posture, vol. 39, no. 2014, pp. S51-S52, 2014, doi: 10.1016/j.gaitpost.2014.04.071.

[9] A. Moussard, E. Bigand, S. Belleville, and I. Peretz, "Music as a Mnemonic to Learn Gesture Sequences in Normal Aging and Alzheimer's Disease," Front. Hum. Neurosci., vol. 8, no. May, pp. 1-9, 2014, doi: 10.3389/fnhum.2014.00294.

[10] C. Pantev and S. C. Herholz, "Plasticity of the human auditory cortex related to musical training," Neurosci. Biobehav. Rev., vol. 35, no. 10, pp. 2140-2154, 2011, doi: 10.1016/j.neubiorev.2011.06.010.

[11] C. Y. Wan and G. Schlaug, "Music making as a tool for promoting brain plasticity across the life span," Neuroscientist, vol. 16, no. 5, pp. 566-577, 2010, doi: $10.1177 / 1073858410377805$.

[12] G. Rosati, A. Rodà, F. Avanzini, and S. Masiero, "On the Role of Auditory Feedback in Robot-Assisted Movement Training after Stroke: Review of the Literature," Comput. Intell. Neurosci., vol. 2013, no. 1-2, pp. 1-15, 2013, doi: 10.1155/2013/586138.

[13] A. J. Sihvonen, T. Särkämö, V. Leo, M. Tervaniemi, E. Altenmüller, and S. Soinila, "Music-based interventions in neurological rehabilitation," Lancet Neurol., vol. 16, no. 8, pp. 648-660, 2017, doi: 10.1016/S1474-4422(17)30168-0.

[14] Y. Tong et al., "Music-supported therapy (MST) in improving post-stroke patients' upper-limb motor function: a randomised controlled pilot study," Neurol. Res., vol. 37, no. 5, pp. 434-440, 2015, doi: 10.1179/1743132815y.0000000034.

[15] T. Särkämö et al., "Music and speech listening enhance the recovery of early sensory processing after stroke," J. Cogn. Neurosci., vol. 22, no. 12, pp. 2716-2727, 2010, doi: 10.1162/jocn.2009.21376.

[16] A. Forsblom, T. Särkämö, S. Laitinen, and M. Tervaniemi, "The Effect of Music and Audiobook Listening on People Recovering From Stroke: The Patient's Point of View," Music Med., vol. 2, no. 4, pp. 229-234, 2010, doi: 10.1177/1943862110378110.

[17] H. G. Hoffman, A. Hollander, K. Schroder, S. Rousseau, and T. Furness, "Physically touching and tasting virtual objects enhances the realism of virtual experiences," Virtual Real., vol. 3, no. 4, pp. 226-234, 1998, doi: 10.1007/BF01408703.

[18] B. Weber, M. Sagardia, T. Hulin, and C. Preusche, "Visual, Vibrotactile, and Force Feedback of Collisions in Virtual Environments: Effects on Performance, Mental Workload and Spatial Orientation," in Shumaker R. (eds) Virtual Augmented and Mixed Reality. Designing and Developing Augmented and Virtual Environments, 2013, pp. 241-250.

[19] C. Ramírez-Fernández, A. L. Morán, and E. García-Canseco, "Haptic Feedback in Motor Hand Virtual Therapy Increases Precision and Generates Less Mental Workload," Proc. 9th Int. Conf. Pervasive Comput. Technol. Healthc., no. July, 2015, doi: 10.4108/icst.pervasivehealth.2015.260242.

[20] H. Sveistrup, "Motor rehabilitation using virtual reality," $J$. Neuroeng. Rehabil., vol. 8, no. January 2005, pp. 1-8, 2004, doi: 10.1186/1743-0003-1-10.
[21] C. Y. Shing, C. P. Fung, T. Y. Chuang, I. W. Penn, and J. L. Doong, "The study of auditory and haptic signals in a virtual reality-based hand rehabilitation system," Robotica, vol. 21, no. 2, pp. 211-218, 2003, doi: 10.1017/S0263574702004708.

[22] N. Barrett, I. Swain, C. Gatzidis, and C. Mecheraoui, "The use and effect of video game design theory in the creation of gamebased systems for upper limb stroke rehabilitation," J. Rehabil. Assist. Technol. Eng., vol. 3, p. 205566831664364, 2016, doi: $10.1177 / 2055668316643644$.

[23] M. Widmer, J. P. Held, F. Wittmann, O. Lambercy, K. Lutz, and A. R. Luft, "Does motivation matter in upper-limb rehabilitation after stroke? ArmeoSenso-Reward: Study protocol for a randomized controlled trial," Trials, vol. 18, no. 1, pp. 1-9, 2017, doi: 10.1186/s13063-017-2328-2.

[24] S. K. Sugawara, S. Tanaka, S. Okazaki, K. Watanabe, and N. Sadato, "Social Rewards Enhance Offline Improvements in Motor Skill," PLoS One, vol. 7, no. 11, p. e48174, Nov. 2012, doi: 10.1371/journal.pone.0048174

[25] G. Richter, D. R. Raban, and S. Rafaeli, "Studying Gamification: The Effect of Rewards and Incentives on Motivation," in Gamification in Education and Business, no. December, T. Reiners and L. Wood, Eds. Cham: Springer International Publishing, 2015, pp. 21-46.

[26] B. Thon, "Cognition and motor skill learning," Ann. Phys. Rehabil. Med., vol. 58, p. e25, Sep. 2015, doi: 10.1016/j.rehab.2015.07.062.

[27] T. Massetti et al., "The Clinical Utility of Virtual Reality in Neurorehabilitation: A Systematic Review," J. Cent. Nerv. Syst. Dis., vol. 10, p. 117957351881354, 2018, doi: $10.1177 / 1179573518813541$.

[28] J. Bai and A. Song, "Development of a Novel Home Based Multi-Scene Upper Limb Rehabilitation Training and Evaluation System for Post-Stroke Patients," IEEE Access, vol. 7, pp. 96679677, 2019, doi: 10.1109/ACCESS.2019.2891606.

[29] M. Ghassemi, J. M. Ochoa, N. Yuan, D. Tsoupikova, and D. Kamper, "Development of an Integrated Actuated Hand Orthosis and Virtual Reality System for Home-Based Rehabilitation," Proc. Annu. Int. Conf. IEEE Eng. Med. Biol. Soc. EMBS, vol. 2018-July, pp. 1689-1692, 2018, doi: 10.1109/EMBC.2018.8512704.

[30] N. B. Pham, V. T. Phan, V. B. Nguyen, and V. D. Nguyen, "Game-based virtual rehabilitation system for upper extremity using low-cost camera," BMEiCON 2015 - 8th Biomed. Eng. Int. Conf., 2016, doi: 10.1109/BMEiCON.2015.7399505. 